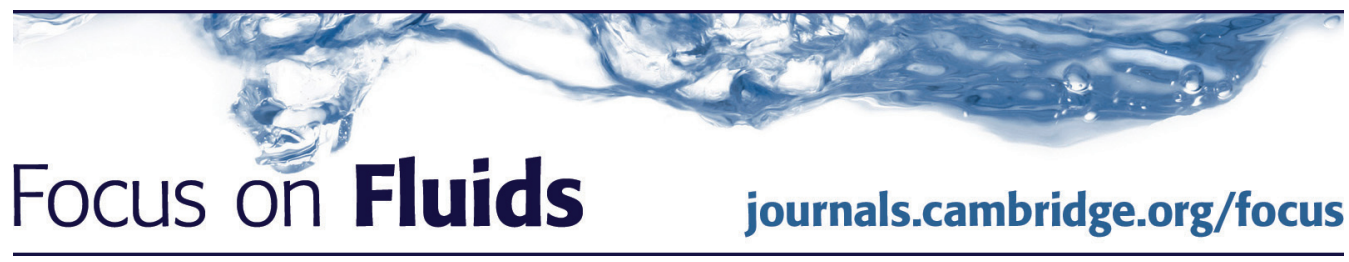

\title{
Growth and breakdown of wave packets in a high-speed boundary layer
}

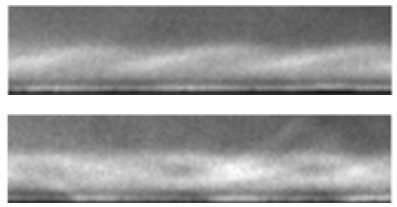

\author{
Aleksandr N. Shiplyuk $\dagger$ \\ Khristianovich Institute of Theoretical and Applied Mechanics, \\ SB RAS, Institutskaya str. 4/1, Novosibirsk 630090, Russia
}

The recent study of Laurence et al. (J. Fluid Mech., vol. 797, 2016, pp. 471-503) develops a new Schlieren-based technique for investigating instabilities and transition in hypersonic boundary layers. This method enables pioneering measurements in a reflected-shock wind tunnel of the characteristics of the second mode of instability on a slender cone, within very short time scales (approximately $1 \mathrm{~ms}$ ). The visualization technique was shown to resolve the structural evolution of individual wave packets. It was revealed that the disturbance strength concentrates near the wall for high-enthalpy conditions.

Key words: boundary-layer stability, high-speed flow

\section{Introduction}

The transition to turbulence of the boundary layers on hypersonic vehicles greatly increases the surface heating rates and frictional drag. It can also considerably decrease the payload capacity for aerospace systems, and increases the weight of thermal protection for reentry spacecrafts. Understanding and predicting the boundary layer transition process is thus essential for hypersonic flight.

It is now well known that empirical correlations of transition locations from wind tunnel experiments are inadequate for extrapolation to flight conditions because of high free-stream disturbance levels in conventional ground-test facilities by comparison with flight (Reshotko 1976), and that the boundary-layer instabilities leading to transition must be measured to make meaningful progress. For hypersonic flows over slender geometries at small angles of attack, the dominant instability is the second mode. Physically, the second-mode disturbances are acoustic waves trapped inside the boundary layer. Their phase speed is about $1-1 / M_{e}$ of the boundary-layer edge velocity (subscript $e$ denotes conditions at the edge of the boundary layer), and their

$\dagger$ Email address for correspondence: shiplyuk@itam.nsc.ru 
wavelength is about two boundary-layer thicknesses, resulting in high frequencies of dominant waves ranging from around $100 \mathrm{kHz}$ to over $1 \mathrm{MHz}$.

Among available experimental data on stability, most have been obtained in 'cold' hypersonic wind tunnels. To study stability and transition for real hypersonic flight conditions, an accompanying real gas with dissociation, chemistry and plasma effects should be simulated. Such a simulation is possible at ground conditions in high-enthalpy, hypervelocity impulse facilities such as reflected-shock wind tunnels and expansion tubes. Test conditions in these facilities offer very short test times (of a few milliseconds) and harsh flow environments (high enthalpy of the order of $10 \mathrm{MJ} \mathrm{kg}^{-1}$, as well as dust and particles in the gas flow). This compounds the difficulties in making instability and transition measurements in hypervelocity tunnels compared to cold hypersonic tunnels. Less than 15 years ago (see e.g. Rasheed et al. 2002) the state of the art for transition measurements in such tunnels was mean heat-flux distributions (through surface-mounted thermocouples) and single-frame shot shadowgraphy. Rapid recent advances in sensor technology and optical methods have enabled the dramatic development of measurement techniques over the past few years, which now allow the direct study of instabilities (e.g. Bountin et al. 2015; Parziale, Shepherd \& Hornung 2015).

The recent work of Laurence, Wagner \& Hannemann (2016), hereafter LWH, develops the Schlieren-based techniques for investigating the second-mode instability and transition in hypersonic boundary layers, and reports pioneering measurements of the characteristics of the instability second mode at high Mach numbers and high enthalpies.

\section{Overview}

The experiments were carried out in the HEG (High Enthalpy shock tunnel Goettingen) of the German Aerospace Center (DLR). The investigation included both relatively low-enthalpy conditions $\left(h_{0}=3.1-3.3 \mathrm{MJ} \mathrm{kg}^{-1}, M_{\infty}=7.34-7.43, u_{\infty}=\right.$ 2420-2360 $\mathrm{m} \mathrm{s}^{-1}$; quasi-steady conditions for $\approx 3 \mathrm{~ms}$ ) and a single high-enthalpy condition $\left(h_{0}=11.9 \mathrm{MJ} \mathrm{kg}^{-1}, M_{\infty}=6.09, u_{\infty}=4400 \mathrm{~m} \mathrm{~s}^{-1}\right.$; quasi-steady conditions for $0.8 \mathrm{~ms}$ ). The model for this study was a slender $7^{\circ}$ half-angle cone with a length of $1100 \mathrm{~mm}$ and a blunt nose of $2.5 \mathrm{~mm}$ radius. A conventional Schlieren arrangement was employed. Density gradients approximately normal to the cone surface were visualized; correspondingly the disturbances of these density gradients (hereafter disturbances) were studied and analysed in the paper. Bursting of the light source (pulses of 20-40 ns duration separated by $2-5 \mu \mathrm{s}$ ), similarly to particle image velocimetry, allowed them to overcome the limitation that the camera frame rate was much lower than the dominant frequency of the instability. Importantly, this allowed the determination of the lengthwise phase velocity of disturbances through spatial image correlation techniques, which meant that frequencies could be measured through transforming what is usually a temporal problem into a spatial one; this in turn enabled the measurement of frequency content well above what traditional surfacemounted sensors are capable of (well over $1 \mathrm{MHz}$ ). The image correlation technique was also applied to determine the structure angle of the disturbances in the boundary layer (the inclination of the constant phase line in streamwise wall-normal plane).

For relatively low enthalpy, LWH investigated the developing second-mode wave packets in the later laminar and early transitional stages of the boundary layer. Time-resolved power spectra show the second-mode wave packets as intermittent concentrations of energy at around $300-400 \mathrm{kHz}$. The resulting value of the mean 
(a)

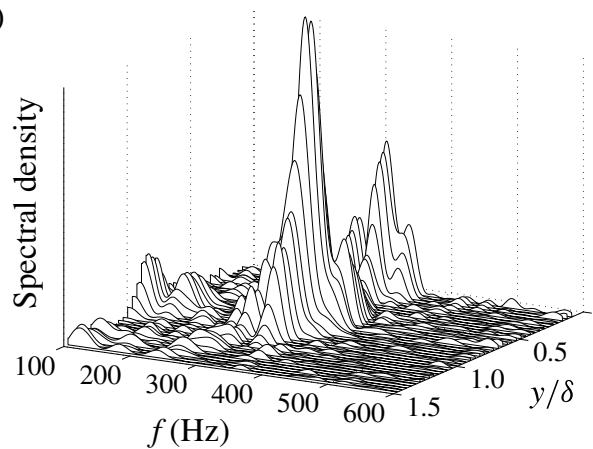

(b)

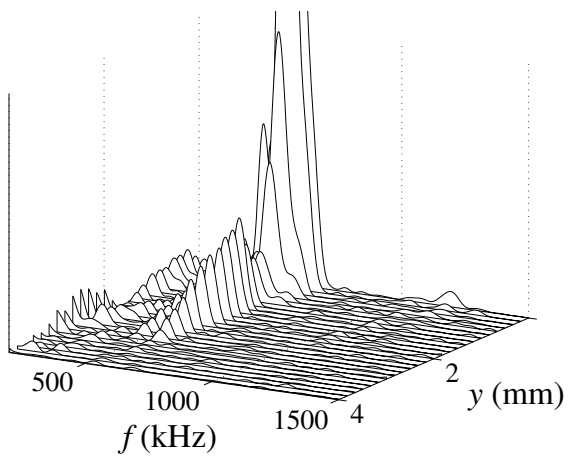

FIgURE 1. Power spectra at different heights above the cone surface for $(a)$ low and

(b) high-enthalpy conditions (from Laurence et al. (2016), figures 12 and 23).

phase speed of boundary layer disturbances is $u_{\text {phase }}=2040-2100 \mathrm{~m} \mathrm{~s}^{-1}$; the rated phase speed is $u_{\text {phase }} / u_{e}=0.85-0.88$. From time to time, the Schlieren images clearly depict distinct wave packets, which first have a rope-like structure (in the upper figure by the title) with two peaks of similar amplitude at the second-mode frequency, located near the wall and in the external part of the boundary layer $(y / \delta \approx 0.7-0.75$, where $y$ is wall-normal distance and $\delta$ is boundary layer thickness), respectively (figure $1 a$ ). The structure angle at the fundamental frequency of the second mode (hereafter fundamental structure angle) decreases from $\sim 90^{\circ}$ near the wall to $10^{\circ}-14^{\circ}$ at $y / \delta \approx 0.8$. As the second-mode amplitude increases, nonlinear interactions lead to first harmonics with a similar two-peak distribution of disturbances. Along with the development of the wave packet further downstream and its approach towards breakdown, the peaks of the rope-like structures appear to fold over, creating a more interwoven appearance reminiscent of plaits or braids (plaited state, the lower figure by the title); a third disturbance peak appears near the external boundary of the boundary layer. The fundamental structure angle undergoes the following changes. In the region between the two original maxima $(y / \delta=0.2-0.4)$, the fundamental structure angle has reached values up to $160^{\circ}$ and gradually the angle close to the $y / \delta=0.7$ maximum increases as the folding over of the wave structures becomes more pronounced. The structure of the wave packet then quickly becomes three-dimensional due to developing nonlinear processes (Bountin, Shiplyuk \& Maslov 2008), which the used Schlieren technique is not able to visualize, so that the packet becomes less distinct.

At high enthalpy, LWH investigated the laminar boundary layer and observed the second-mode wave packets at frequencies of approximately $600 \mathrm{kHz}$, phase velocity $u_{\text {phase }} \approx 3680-3810 \mathrm{~m} \mathrm{~s}^{-1}$ (or $u_{\text {phase }} / u_{e}=0.85-0.88$ ), with a very weak first harmonic at $1200 \mathrm{kHz}$. The disturbances amplitude distribution across to the boundary layer was modified compared to the low-enthalpy case. The two-peak distribution was also observed, but the disturbance strength near the wall was much higher than anywhere else inside the boundary layer (figure $1 b$ ).

\section{Future}

The laminar-turbulent transition in boundary layers at hypersonic flight velocities is a very delicate and complicated process in which many instability mechanisms compete, and which is influenced by every physicochemical effect in the flow. The work of LWH enables advancement into the area of the high-enthalpy processes typical of high flight velocities like $M=8-14$ and to gather new data on the wave 
packet development under these conditions. LWH were the first to demonstrate the difference in the wave packet development for the low- and high-enthalpy cases. In the latter case, the main part of the disturbances concentrates near the wall.

The technique developed in LWH is ideally suited to testing important recent theoretical predictions regarding the radiation of sound by wave packets (Chuvakhov \& Fedorov 2016). It would be particularly fruitful to combine the technique with lateral arrays of sensors (e.g. Casper, Beresh \& Schneider 2014) to gain a more complete picture of wave-packet development in all dimensions. Indeed, in the non-focusing Schlieren arrangement used by LWH, the measurements integrate the density gradients along the line of sight. This is appropriate for natural second-mode disturbances, since they are two-dimensional (Fedorov et al. 2006), but ensemble-averaged wall-normal distributions on cones and three-dimensional disturbances will not be reliably resolved by the line-of-sight technique. The technique could also be developed further to allow calibration, so that quantitative data on disturbance amplitudes may be obtained. This will allow the measurements of amplification rates that can be compared quantitatively to calculations. Finally, it would be interesting to develop an artificial wave-packet technique with generation of artificial disturbances of prescribed frequency and phase for hypervelocity tunnels in order to increase the accuracy of wave characteristics measurements (e.g. Lyttle et al. 2005). In conclusion, the novel experimental technique developed by LWH provides a new baseline for experimental research on the stability of hypervelocity boundary layers, from which further developments are likely to emerge.

\section{References}

Bountin, D. A., Gromyko, Yu. V., Maslov, A. A., Polivanov, P. A. \& Sidorenko, A. A. 2015 On the determination of the position of laminar-turbulent transition in boundary layer by optical methods. Thermophys. Aeromech. 22 (6), 767-770.

Bountin, D. A., Shiplyuk, A. N. \& Maslov, A. A. 2008 Evolution of nonlinear processes in a hypersonic boundary layer on a sharp cone. J. Fluid Mech. 611, 427-442.

CAsper, K. M., Beresh, S. J. \& SChNeIDer, S. P. 2014 Pressure fluctuations beneath instability wavepackets and turbulent spots in a hypersonic boundary layer. J. Fluid Mech. 756, $1058-1091$.

Chuvakhov, P. V. \& Fedorov, A. V. 2016 Spontaneous radiation of sound by instability of a highly cooled hypersonic boundary layer. AIAA Paper 2016-4245.

Fedorov, A. V., Kozlov, V. F., Shiplyuk, A. N., Maslov, A. A. \& Malmuth, N. D. 2006 Stability of hypersonic boundary layer on porous wall with regular microstructure. AIAA $J$. 44 (8), 1866-1871.

Fedorov, A. V., Shiplyuk, A. N., Maslov, A. A., Burov, E. V. \& Malmuth, N. 2003 Stabilization of a hypersonic boundary layer using an ultrasonically absorptive coating. J. Fluid Mech. 479, 99-124.

Laurence, S. J., Wagner, A. \& Hannemann, K. 2014 Schlieren-based techniques for investigating instability and transition in a hypersonic boundary layer. Exp. Fluids 55, 1782.

Laurence, S. J., Wagner, A. \& Hannemann, K. 2016 Experimental study of second-mode instability growth and breakdown in a hypersonic boundary layer using high-speed Schlieren visualization. J. Fluid Mech. 797, 471-503.

Lyttle, I. J., Reed, H. L., Shiplyuk, A. N., Maslov, A. A., Buntin, D. A. \& Schneider, S. P. 2005 Numerical-experimental comparisons of second-mode behavior for blunted cones. AIAA J. 43 (8), 1734-1743.

Parziale, N. J., Shepherd, J. E. \& Hornung, H. G. 2015 Observations of hypervelocity boundary-layer instability. J. Fluid Mech. 781, 87-112.

Rasheed, A., Hornung, H. G., Fedorov, A. V. \& Malmuth, N. D. 2002 Experiments on passive hypervelocity boundary layer control using an ultrasonically absorptive surface.. AIAA $J$. 40 (3), 481-489.

Reshotко, E. 1976 Boundary-layer stability and transition. Annu. Rev. Fluid Mech. 8, 311-349. 